\title{
ISG15 inhibits cancer cell growth and promotes apoptosis
}

\author{
MEI-JUAN ZHOU ${ }^{1 *}$, FANG-ZHI CHEN ${ }^{2 *}$, HAN-CHUN CHEN ${ }^{1}$, XIN-XING WAN ${ }^{1}$, \\ $\mathrm{XI} \mathrm{ZHOU}^{1}$, QIAN FANG ${ }^{1}$ and DIAN-ZHENG ZHANG ${ }^{1,3}$
}

\author{
${ }^{1}$ Department of Biochemistry, School of Life Sciences and the State Key Laboratory of Medical Genetics, \\ Central South University, Changsha, Hunan 410013; ${ }^{2}$ Department of Urology, The Second Xiangya Hospital of \\ Central South University, Changsha, Hunan 410011, P.R. China; ${ }^{3}$ Department of Bio-Medical Sciences, \\ Philadelphia College of Osteopathic Medicine, Philadelphia, PA 19131, USA
}

Received December 25, 2015; Accepted December 20, 2016

DOI: $10.3892 /$ ijmm.2016.2845

\begin{abstract}
Cervical cancer is one of the most common causes of cancer-related mortality in women in developing countries. Interferon (IFN)- $\alpha$ has been widely used in the treatment of various types of cancer, including cervical cancer, and IFN-stimulated gene 15 (ISG15), an ubiquitin-like protein, is upregulated by IFN- $\alpha$ treatment. The anti-virus and antitumor effects of ISG15 have been reported; however, its mechanism of action have not yet been fully elucidated. In this study, HeLa cells were used as a model system to investigate the roles of ISG15 in IFN- $\alpha$-mediated cancer cell growth inhibition and induction of apoptosis. The results revealed that both p53 and p21 were upregulated in HeLa cells treated with IFN- $\alpha$ or in the HeLa cells overexpressing ISG15. In addition, the expression levels of ubiquitin-like modifier-activating enzyme 7 (UBA7, also known as UBE1L; ISG15 E1-activating enzyme), UBCH8 (ISG15 E2-conjugating enzyme) and HERC5 (ISG15 E3-ligase) were elevated in the HeLa cells treated with IFN- $\alpha$. The levels of p53 in the HeLa cells were attenuated by transient transfection with small interfering RNA (siRNA) targeting ISG15 (ISG15-siRNA). Cell viability was inhibited by both IFN- $\alpha$ treatment and ISG15 overexpression. However, these effects were significantly diminished when p53 was knocked down, suggesting that the effects of inhibitory effects of ISG15 on HeLa cell growth and the induction of apoptosis were p53-dependent. Taken together, these results suggest the existence of the IFN- $\alpha /$ ISG15/p53 axis in cervical cancer cells and any strategies manipulating the levels
\end{abstract}

Correspondence to: Professor Han-Chun Chen, Department of Biochemistry, School of Life Sciences and the State Key Laboratory of Medical Genetics, Central South University, 172 Tongzipo Road, Changsha, Hunan 410013, P.R. China

E-mail: chenhanchun@csu.edu.cn

*Contributed equally

Key words: HeLa cells, interferon-stimulated gene 15, interferon- $\alpha$, p53, UBE1L, UBCH8, HERC5 of ISG15 may thus prove to be effective in the treatment of cervical cancer.

\section{Introduction}

Cervical cancer is the third most commonly diagnosed cancer after breast and colorectal cancers and one of the most common causes of cancer-related mortality in women in developing countries (1). Infection with human papillomavirus (HPV) is an important contributing factor in the development of cervical cancer. Mechanistically, the insertion of viral DNA into chromosomal DNA results in either the activation of protooncogenes or the inactivation of tumor suppressor genes, which leads to uncontrolled cell proliferation and cervical intraepithelial neoplasia (CIN) (2). Eventually, approximately $30 \%$ of all CIN cases will develop into cervical cancer (3). Previous studies using different cancer cell models, including $\mathrm{HeLa}$ cells have demonstrated that interferon (IFN)- $\alpha$ is capable of inhibiting cancer cell proliferation and inducing apoptosis (4). In addition, IFNs has also been reported to be used in cervical cancer treatment (5). However, the efficacy of IFN in cervical cancer therapy has proven to be inconsistent and sometimes controversial (6). Moreover, resistance to IFNs has also been observed in the treatment of certain types of HPV-associated cervical cancers (7). Understanding the mechanisms responsible for the effects of IFN- $\alpha$ on cancer cells may aid in the clinical application of IFN- $\alpha$ in cervical cancer therapies.

Type I interferons (IFN- $\alpha$ and IFN- $\beta$ ), a family of secreted proteins, were originally identified as cytokines with important antiviral properties (8), and therefore have been widely used as important anti-viral drugs since their discovery in 1957 (9). Mechanistically, they bind their specific receptors (IFNAR1 and IFNAR2) presenting on the cell surface and activate Janus-activated kinase (JAK)/signal transducer and activator of transcription (STAT) signaling pathways, activating different cellular cascades, including antiviral defense and immune modulation (10). At the molecular level, IFNs initiate a genetic program that orchestrates the regulation of hundreds of genes known as IFN-stimulated genes (ISGs). Previous studies have suggested that IFNs may also possess anticancer functions by inhibiting cancer cell growth and inducing apoptosis $(11,12)$.

IFN-stimulated gene 15 (ISG15), an ubiquitin-like protein, is highly upregulated by IFNs (13). It is initially translated as 
a 165 amino acids precursor and is subsequently processed to expose its functional C-terminal sequence, LRLRGG. ISG15 contains two ubiquitin-like domains with the $\mathrm{N}$ - and C-terminal domain bearing 33 and $32 \%$ identity to that of ubiquitin, respectively $(13,14)$. Also like ubiquitin, ISG15 exists either as a free moiety or is conjugated to a myriad of proteins (15). Since ISG15 was originally found to cross-react with certain ubiquitin antibodies, it has been also known as ubiquitin cross-reactive protein (16). The conjugation of ISG15 to target proteins is processed through three consecutive steps, including activation, conjugation and ligation. This process is widely known as ISGylation, involving at least three classes of enzymes: the E1 activating enzyme UBE1L, the E2 conjugating enzymes UBCH8 and UBCH6, and the E3 ligases EFP and HERC5, which are all regulated by IFNs $(8,17)$. In addition, the identification of the deconjugating enzyme ISG15-specific protease USP18 (UBP43) provides an explanation for the dynamic ISGylation status of a particular protein. Functionally, ISGylation can either activate or inhibit the activity of a specific target protein (18). Nevertheless, unconjugated free ISG15 itself also possesses antiviral activities (19).

Significant efforts have been made to identify the substrates of ISG15 conjugation and their roles in pathogen infection and tumorigenesis $(20,21)$. In this study, HeLa cells were used as a model system to examine the roles of ISG15 in cervical cancer. ISG15 expression was found to be increased in HeLa cells treated with IFN- $\alpha$ or transiently transfected with ISG15 overexpression plasmid. Both p53 and p21 were also upregulated when the HeLa cells were either treated with IFN- $\alpha$ or transiently transfected with ISG15 overexpression plasmid. Our study reveals the existence of the IFN- $\alpha / \mathrm{ISG} 15 / \mathrm{p} 53$ axis in cervical cancer cells, and suggest that manipulating ISG15 expression may prove to be effective in the treatment of cervical cancer.

\section{Materials and methods}

Cell culture and IFN- $\alpha$ treatment. HeLa cells (obtained from Department of Molecular Biology, Central South University, Changsha, China) were grown in DMEM (Gibco, Grand Island, NY, USA) supplemented with $10 \%$ fetal bovine serum (Biological Industries, Beit Haemek, Israel), $100 \mathrm{U} /$ $\mathrm{ml}$ of penicillin and $100 \mu \mathrm{g} / \mathrm{ml}$ of streptomycin, at $37^{\circ} \mathrm{C}$ in an atmosphere with $5 \% \mathrm{CO}_{2}$. The HeLa cells were treated with IFN- $\alpha$ (Sigma-Aldrich, St. Louis, MO, USA) at various concentrations $(0,500,1,000$ and 2,000 $\mathrm{U} / \mathrm{ml})$ for $48 \mathrm{~h}$ in 6 -well plates.

Plasmid transfection. The PCDNA3.1 and PCDNA3.1-ISG15 plasmids were previously constructed in our laboratory (22). The cells were seeded in 6 -well plates $\left(8 \times 10^{5}\right.$ cells/well $)$ and transfected with Lipofectamine 2000 transfection reagent (Invitrogen Life Technologies, Carlsbad, CA, USA) in accordance with the manufacturer's instructions. The transfected cells were then cultured in DMEM supplemented with $10 \%$ fetal bovine serum for $48 \mathrm{~h}$ for use in further experiments as described below.

3-(4,5-Dimethyl-2-thiazolyl)-2,5-diphenyltetrazolium bromide (MTT) assay. The cells $\left(10^{4}\right.$ cells/well) were plated in 96-well plates. MTT assays were conducted following the different treatments. The cells were incubated with MTT for $4 \mathrm{~h}$ at $37^{\circ} \mathrm{C}$.
The dye produced by viable cells was dissolved in DMSO and the absorbance values were measured at $490 \mathrm{~nm}$ using an EnSpire Multilabel Reader (Perkin Elmer Singapore Pte. Ltd., Singapore). All assays were performed at least 3 times.

RNA interference. Specific small interfering RNA (siRNA) targeting ISG15 (AY168648; ISG15-siRNA) (5'-UGAGC ACCGUGUUCAUGAATT-3', 5'-UUCAUGAACACGGUGC UCATT-3'), p53 (AB082923) (5'-CCACCAUCCACUACAA CUATT-3', 5'-UAGUUGUAGUGGAUGGUGGTT-3'), and scrambled siRNA (5'-UUCUCCGAACGUGUCACGUTT-3', 5'-ACGUGACACGUUCGGAGAATT-3') were obtained from GenePharm (Shanghai, China). The cells $\left(5 \times 10^{5} /\right.$ well) were transfected with 100 pmol siRNA using Lipofectamine 2000 transfection reagent in accordance with the manufacturer's instructions. Following culture for $48 \mathrm{~h}$ post-transfection, the cells were collected for use in further experiments.

Western blot analysis. The cells were harvested and lysed with cold RIPA buffer (Beyotime Institute of Biotechnology, Inc., Shanghai, China) supplemented with $1 \mathrm{mM}$ proteinase inhibitor PMSF (Solarbio, Beijing, China) for $30 \mathrm{~min}$ on ice, and then centrifuged at $12,000 \mathrm{rpm}$ for $16 \mathrm{~min}$ at $4^{\circ} \mathrm{C}$. The protein concentration was measured using a BCA protein assay kit (Thermo Fisher Scientific, Inc., Waltham, MA, USA). Proteins were first separated by SDS-PAGE and then transferred onto polyvinylidene fluoride (PVDF) membranes (Millipore, Billerica, MA, USA) by electro-blotting and blocked in TBST $(50 \mathrm{mM}$ Tris- $\mathrm{HCl}, \mathrm{pH} 7.5,150 \mathrm{mM} \mathrm{NaCl}$ and $0.2 \%$ Tween-20) containing $5 \%$ non-fat milk for $4 \mathrm{~h}$ at $4{ }^{\circ} \mathrm{C}$. The blots were incubated with primary antibodies $(1: 1,000)$ against the control protein glyceraldehyde 3-phosphate dehydrogenase (GAPDH) (1:1,000; Cat. no. 10494-1-AP), rabbit anti-p53 (1:1,000; Cat. no. 10442-1-AP), rabbit anti-p21 (1:1,000; Cat. no. 10355-1-AP), rabbit anti-UBE1L (1:500; Cat no. 15818-1-AP), rabbit anti-UBCH8 (1:500; Cat. no. 11844-1-AP) and rabbit anti-HERC5 (1:500; Cat. no. 22692-1-AP) (all from Proteintech Group, Inc., Chicago, IL, USA), rabbit anti-ISG15 (1:1,000; Cat. no. 2743; Cell Signaling Technology,Inc., Boston, MA, USA) at $4^{\circ} \mathrm{C}$ overnight and washed 3 times with TBST. The blots were then incubated with the secondary antibody $(1: 1,000)$ at room temperature for $1 \mathrm{~h}$. The horseradish peroxidase (HRP)-conjugated secondary antibody was purchased from Beyotime Institute of Biotechnology, Inc.

Flow cytometry and caspase-3 activity detection. After being washed with PBS ( $\mathrm{pH} 7.4)$, the cells were suspended in binding buffer and Annexin V-FITC and DNA dye propidium iodide (PI) were added followed by incubation at $37^{\circ} \mathrm{C}$ for $30 \mathrm{~min}$ in a dark room. The cells were analyzed by Well Biotechnology, Co., Ltd. (China) using Cytomics FC500 (Beckman Coulter, Brea, CA, USA). Caspase-3 activity was determined using the caspase-3 activity assay kit (Beyotime Insititute of Biotechnology, Inc.). Following treatment, the 96-well plate was incubated for $1 \mathrm{~h}$ at $37^{\circ} \mathrm{C}$. The absorbance values were measured at $405 \mathrm{~nm}$ using an EnSpire Multilabel Reader (Perkin Elmer Singapore Pte. Ltd.).

Statistical analysis. Data were analyzed by one-way analysis of variance (ANOVA) and LSD using SPSS 16.0 
A

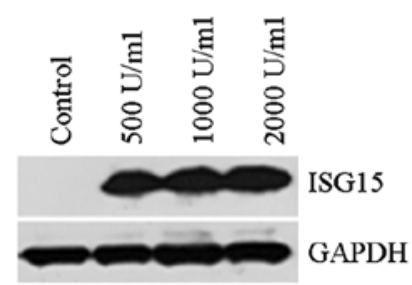

$\mathrm{C}$

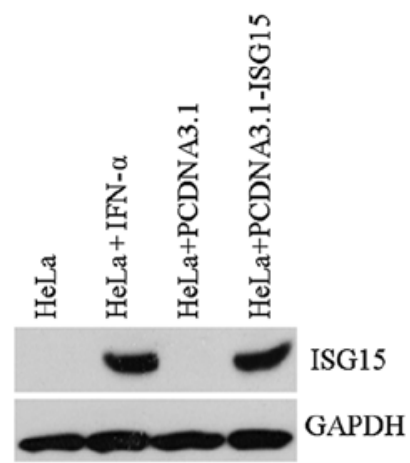

B

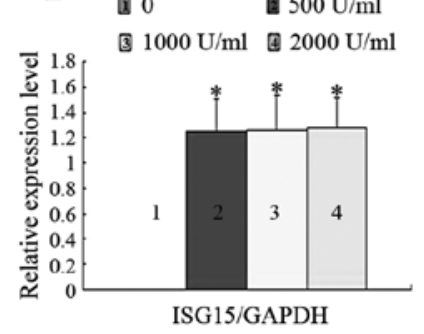

$\mathrm{D}$

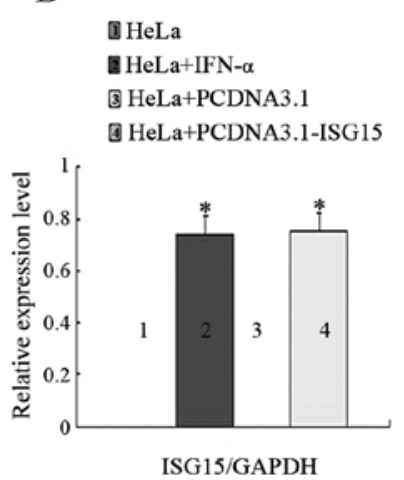

A.

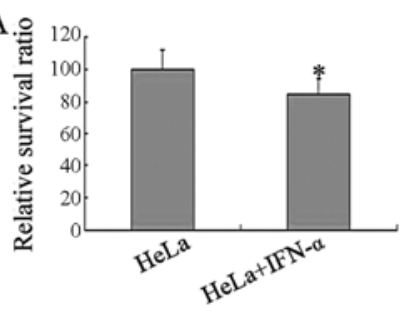

$\mathrm{C}$

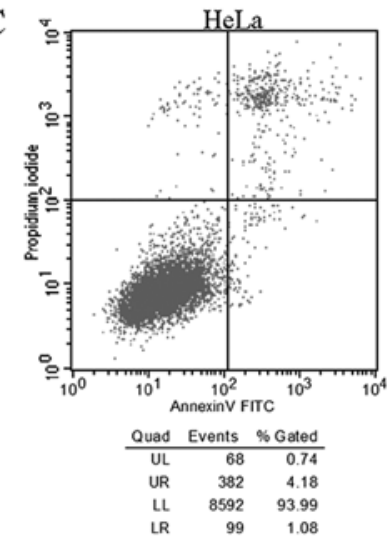

$\mathrm{E}$

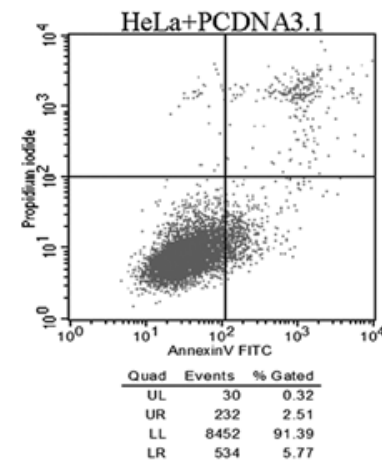

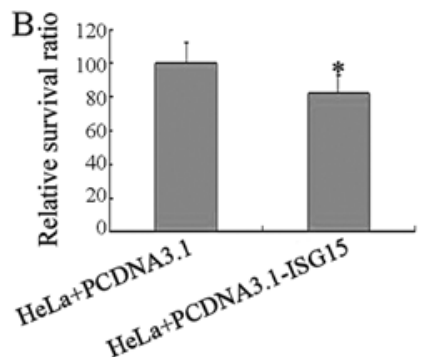

$\mathrm{D}$

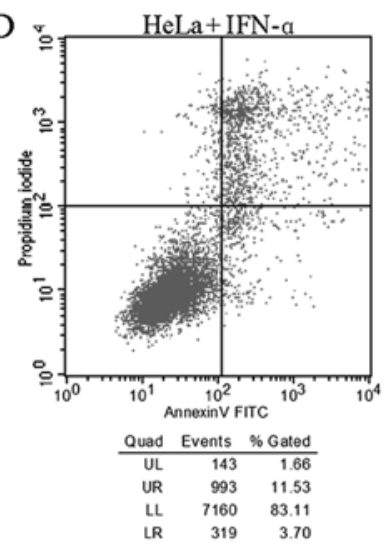

$\mathrm{F}$

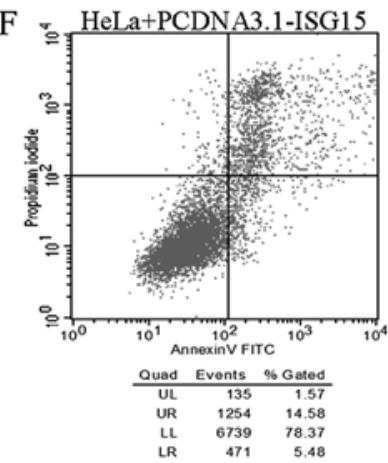

cells. (A) HeLa cells were treated with various concentrations of IFN- $\alpha$ for 48 h. (C) HeLa cells treated with IFN- $\alpha$ or transiently transfected with differen plasmids were cultured for $48 \mathrm{~h}$. (B and D) Densities of the western blot bands were quantified using Image J software (gray scale band analysis) and plotted. Results are shown as the means \pm SD $(n=3)$. * $P \leq 0.05$ indicates significant difference compared to the control.

software (SPSS, Inc., Chicago, IL, USA). Densitometric analysis of the western blot bands was carried out using Image J software. The results were presented as the means \pm standard deviation (SD). Differences were considered significant if the $\mathrm{P}$-value was $\leq 0.05$.

\section{Results}

Expression and induction of ISG15 by IFN- $\alpha$. The cells were treated with various concentrations of IFN- $\alpha$ for $48 \mathrm{~h}$, and ISG15 expression was detected by western blot analysis. As shown in Fig. 1A and B, ISG15 was undetectable in the untreated control cells, and its expression level was increased in response to IFN- $\alpha$ treatment, although a statistically significant concentration-dependent effect $(500 \mathrm{U} / \mathrm{ml} ; 1,000 \mathrm{U} / \mathrm{ml} ; 2,000 \mathrm{U} / \mathrm{ml})$ of IFN- $\alpha$ was not observed. The concentration of $1,000 \mathrm{U} / \mathrm{ml}$ IFN- $\alpha$ was selected for use in the subsequent experiments based on the commonly used dosage in clinical practice. As shown in Fig. 1C and D, the transient transfection of the cells with the empty plasmid vector, PCDNA3.1, had no effect on ISG15 expression. Compared with the control group, the expression levels of ISG15 were increased in response to both IFN- $\alpha$ treatment and transient transfection with PCDNA3.1-ISG15 plasmid.

Effect of ISG15 on cell viability. Cell viability was examined by MTT assay after the cells were treated with IFN- $\alpha$ or transiently transfected with the PCDNA3.1-ISG15 plasmid. As shown in Fig. 2A and B, the viability of the cells was significantly decreased (100 vs. $84.93 \%)$ when they were

Figure 2. Effects of interferon (IFN)-stimulated gene 15 (ISG15) on HeLa cell viability and apoptotic status. (A and B) Viability of the cells was estimated by MTT assays when the cells were treated with IFN- $\alpha(1,000 \mathrm{U} / \mathrm{ml})$ for $48 \mathrm{~h}$ or transiently transfected with PCDNA3.1-ISG15 plasmid. (C-F) Apoptotic status of the cells was analyzed by flow cytometry (single determination). The apoptotic cell scatter plots are divided into 4 quadrants. They are the upper left (UL), upper right (UR), lower left (LL), and lower right (LR) quadrants, which show damaged (necrotic) cells, late apoptotic cells, living cells, and early apoptotic cells, respectively. Results are shown as the means \pm SD $(n=3)$. ${ }^{*} \mathrm{P} \leq 0.05$ indicates significant difference compared to the control.

exposed to $1,000 \mathrm{U} / \mathrm{ml}$ of IFN- $\alpha$, and the viability of the cells decreased from $100 \%$ (the PCDNA3.1 plasmid) to $82.02 \%$ when the cells were transfected with the PCDNA3.1-ISG15 plasmid. These data indicated that IFN- $\alpha$ treatment and ISG15 overexpression have similar effects on HeLa cell viability. The cell apoptotic status was further analyzed by flow cytometry under different conditions. As shown in Fig. 2C-F, the proportion of apoptotic cells was increased by IFN- $\alpha$ treatment or transient transfection with the PCDNA3.1-ISG15 plasmid. The detailed apoptotic data (Table I) revealed that the number of apoptotic cells in both the early and late stages of apoptosis was significantly elevated by IFN- $\alpha$ treatment or ISG15 overexpression. In addition, the caspase- 3 enzymatic activity in the HeLa cells either treated with IFN- $\alpha$ or transiently transfected 
Table I. Effect of IFN- $\alpha$ or ISG15 on HeLa cell apoptosis.

\begin{tabular}{lcc}
\hline Groups & $\begin{array}{c}\text { Early apoptosis } \\
(\%)\end{array}$ & $\begin{array}{c}\text { Late apoptosis } \\
(\%)\end{array}$ \\
\hline HeLa & 1.08 & 4.18 \\
HeLa + IFN- $\alpha$ & 3.70 & 11.53 \\
HeLa + PCDNA3.1 & 5.77 & 2.51 \\
HeLa + PCDNA3.1-ISG15 & 5.48 & 14.58
\end{tabular}

IFN, interferon; ISG15, interferon-stimulated gene 15 .
A

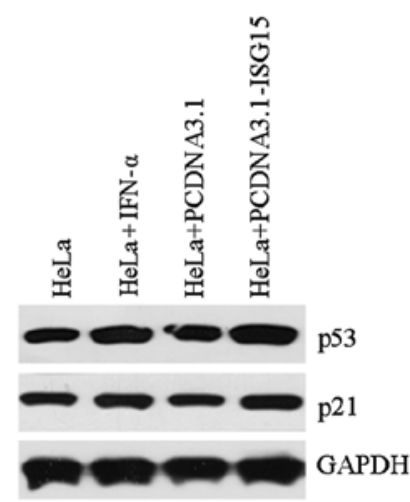

C

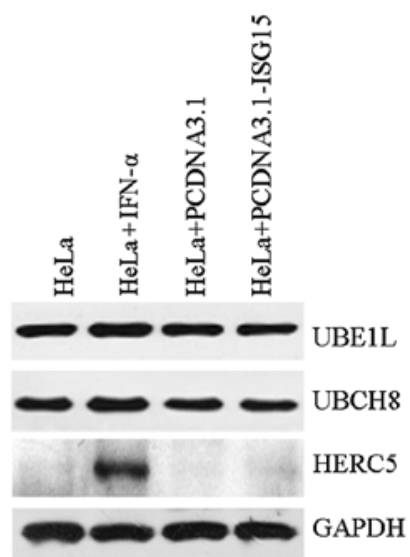

B

[1] HeLa

a HeLa+IFN- $\alpha$ 3) $\mathrm{HeLa}+\mathrm{PCDNA3.1}$ (9) HeLa+PCDNA3.1-ISG15

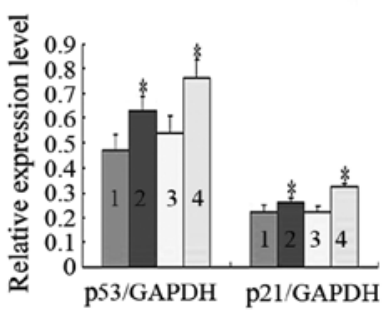

$\mathrm{D}$
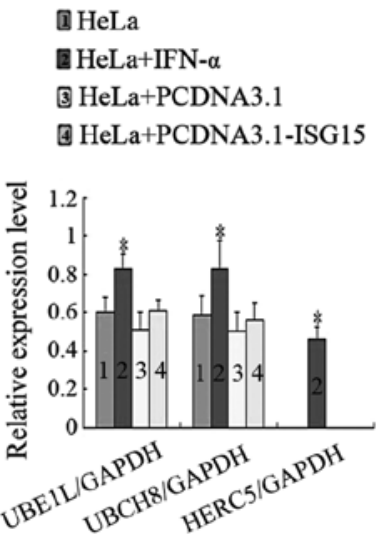

A

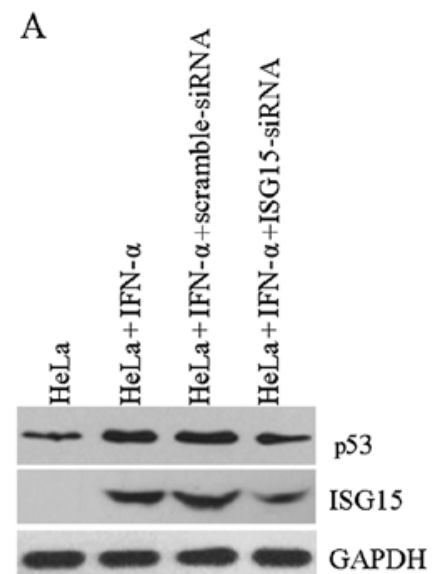

$\mathrm{C}$
B

国 HeLa

通 $\mathrm{HeLa}+\mathrm{IFN}-\alpha$

(3) HeLa+IFN- $\alpha+$ scramble-siRNA

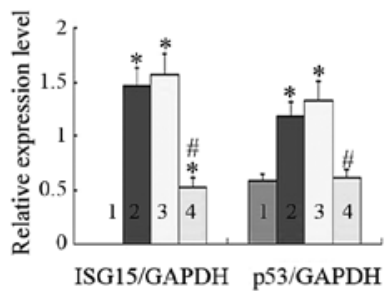

D
QHeLa+IFN- $\alpha+$ ISG15-siRNA

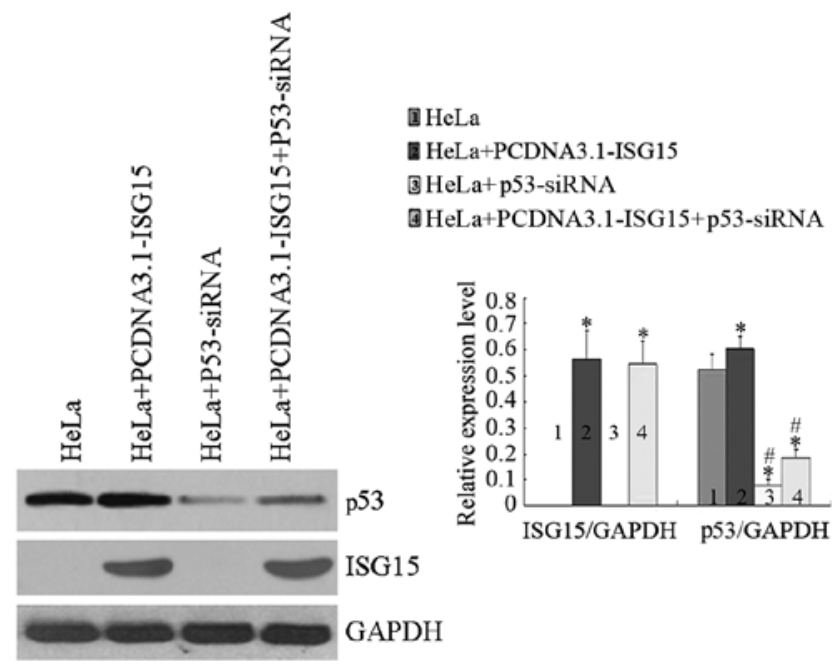

Figure 4. Role of interferon (IFN)-stimulated gene 15 (ISG15) in the regulation of p53 expression. (A) HeLa cells treated without (lane 1, from left to right) or with (lane 2) IFN- $\alpha$ were then transfected with scrambled siRNA (lane 3) or siRNA specifically against ISG15 (lane 4). (C) HeLa cells without (lane 1) or with (lane 2) overexpression of ISG15 or were transfected with siRNA specifically against p53 (lane 3) or overexpression of ISG15 were transfected with siRNA specifically against p53 (lane 4). (B and D) The intensities of the western blot bands were semi-quantified and the ratios between ISG15/ GAPDH and p53/GAPDH were plotted. Results are shown as the means \pm SD $(n=3) . ~ P \leq 0.05$ indicates significant difference compared to the control and ${ }^{\#} \mathrm{P} \leq 0.05$ indicates significant difference compared to the cells treated with IFN- $\alpha$ or transfected with PCDNA3.1-ISG15 plasmid.

Figure 3. Effects of interferon (IFN)-stimulated gene 15 (ISG15) on p53, p21, UBE1L, UBCH8 and HERC5 expression. HeLa cells treated with IFN- $\alpha$ or transiently transfected with ISG15 overexpression plasmid were cultured for $48 \mathrm{~h}$. (A) The levels of p53 and p21 were examined by western blot analysis using anti-p53 and anti-p21 antibodies. (C) The expression levels of UBE1L, UBCH8 and HERC5 were assessed by western blot analysis using anti-UBE1L, anti-UBCH8 and anti-HERC5 antibodies. (B and D) Densities of the western blot bands were quantified using ImageJ software (gray scale band analysis) and plotted. Results are shown as the means \pm SD $(n=3)$. ${ }^{*} \leq 0.05$ indicates significant difference compared to the control.

with the PCDNA3.1-ISG15 plasmid was measured. As shown in Table II, compared with the basal levels in the control HeLa cells, either treatment with IFN- $\alpha$ or transfection with the PCDNA3.1-ISG15 plasmid increased caspase-3 enzymatic activity. These results suggest that the overexpression of ISG15 or treatment with IFN- $\alpha$ play the same important roles in HeLa cell growth inhibition and the induction of apoptosis.
Effects of ISG15 on p53, p21, UBE1L, UBCH8 and HERC5 expression. Since p53 is one of the key tumor suppressors in the regulation of tumor cell survival and apoptosis, the effects of IFN- $\alpha$ treatment or ISG15 overexpression on the expression of p53 and its downstream target, p21, were investigated. The protein levels of p53 and p21 were examined by western blot analysis using specific antibodies. As shown in Fig. 3A, compared to the controls, the levels of p53 and p21 were markedly elevated in the cells treated with IFN- $\alpha$ or transfected with PCDNA3.1-ISG15 plasmid. As shown in Fig. 3C, the expression levels of UBE1L, UBCH8 and HERC5 were upregulated in the cells treated with IFN- $\alpha$. However, the overexpression of ISG15 did not affect the levels of UBE1L, UBCH8 and HERC5 expression. The gray scale protein band intensity from 3 experiments was quantified by ImageJ software (Fig. 3B and D). These 
Table II. Effect of IFN- $\alpha$ or ISG15 on caspase-3 activity in HeLa cells $(n=3)$.

\begin{tabular}{lc} 
Groups & $\begin{array}{c}\text { Caspase-3 enzymatic } \\
\text { activity }(\mathrm{U} / \mu \mathrm{g})\end{array}$ \\
\hline HeLa & $3.13 \pm 1.45$ \\
HeLa + IFN- $\alpha$ & $7.73 \pm 2.08^{\mathrm{a}}$ \\
HeLa + PCDNA3.1 & $3.39 \pm 1.96$ \\
HeLa + PCDNA3.1-ISG15 & $8.37 \pm 2.25^{\mathrm{a}}$ \\
\hline
\end{tabular}

${ }^{a} \mathrm{P} \leq 0.05$ indicates significant difference compared to control. IFN, interferon; ISG15, interferon-stimulated gene 15.

Table III. The relative survival ratio of HeLa cells $(n=3)$.

\begin{tabular}{lc}
\hline Groups & $\begin{array}{c}\text { Relative survival } \\
\text { ratio }(\%)\end{array}$ \\
\hline HeLa & $100.0 \pm 8.4$ \\
HeLa + IFN- $\alpha$ & $87.5 \pm 8.0^{\mathrm{a}}$ \\
HeLa + IFN- $\alpha+$ scramble-siRNA & $88.3 \pm 7.7^{\mathrm{a}}$ \\
HeLa + IFN- $\alpha+$ ISG15-siRNA & $98.8 \pm 8.0$ \\
HeLa + PCDNA3.1-ISG15 & $87.0 \pm 8.7^{\mathrm{a}}$ \\
HeLa + p53-siRNA & $100.0 \pm 7.9$ \\
HeLa + PCDNA3.1-ISG15 + p53-siRNA & $99.5 \pm 7.7$ \\
\hline
\end{tabular}

${ }^{a} \mathrm{P} \leq 0.05$ indicates significant difference compared to control. IFN, interferon; siRNA, small interfering RNA; ISG15, interferon-stimulated gene 15 .

results indicate that free ISG15 is related to inhibition of HeLa cell proliferation.

Knockdown and expression of ISG15 and p53. In order to elucidate the role of ISG15 in the regulation of p53 expression, ISG15 was knocked down by siRNA and the level of p53 was analyzed under different conditions. As shown in Fig. $4 \mathrm{~A}$ and $\mathrm{B}$, transfection of the cells with siRNA against ISG15 downregulated ISG15 by $>50 \%$; however, transfection with scrambled siRNA did not affect the IFN- $\alpha$-induced ISG15 expression. More importantly, IFN- $\alpha$-induced p53 expression was also significantly decreased when ISG15 was knocked down; however, transfection with scrambled siRNA had no effect on the IFN- $\alpha$-induced p53 expression. As shown in Fig. 4C, compared with the untreated controls, the expression of p53 was increased in the cells transfected with the PCDNA3.1-ISG15 plasmid. Transfection with siRNA against p53 was able to knock down both the endogenous, as well as the ISG15-induced p53 expression. Moreover, cell viability was measured by MTT assay when ISG15 and/or p53 were knocked down. As shown in Table III, cell viability was reduced to a similar degree when the cells were either treated with IFN- $\alpha(87.5 \%)$ or transfected with the ISG15 overexpression plasmid $(87 \%)$. However, the effect of IFN- $\alpha$ on cell viability was completely diminished when siRNA against ISG15, but not scrambled siRNA, was transfected into the cells, indicating that ISG15 is essential in the IFN- $\alpha$-mediated reduction of HeLa cell viability. Furthermore, the effect of the overexpression of ISG15 on cell viability was not observed when siRNA against p53, but not scrambled siRNA, was transfected into the cells, suggesting that the above-observed effects of ISG15 are p53-dependent. Taken together, these data demonstrate that p53 plays an essential role in the IFN- $\alpha /$ ISG15-mediated apoptosis of HeLa cells.

\section{Discussion}

Although type I interferons, particularly IFN- $\alpha$, have been used in the treatment of various types of cancer, the precise molecular mechanisms of action of IFNs in cancer treatment are far from being elucidated $(23,24)$, and this severely hinders the further application of IFNs in cancer therapy. One of the most well-studied IFN- $\alpha$ signaling mechanisms is the JAK/ STAT-mediated rapid induction of ISGs $(25,26)$. Among the ISGs, ISG15 is the most important one and it exerts its effects on cancer cells through protein ISGylation via its C-terminal LRLRGG sequence or in the form of a free moiety $(15,27)$. However, the effect of ISGylation on a specific protein could be inhibitive or simulative (18). Since high levels of ISG15 and upregulated ISGylation have been observed in certain types of cancer, it has also been postulated that ISG15 may directly or indirectly be involved in tumor development (28). In this study, the human cervical cancer cell line, HeLa, was used as the model system to demonstrate that IFN-induced ISG15 inhibits cell growth and induces apoptosis in a p53-dependent manner; we therefore revealed the existence of the IFN- $\alpha / \mathrm{ISG} 15 / \mathrm{p} 53$ signaling axis in cervical cancer cells.

In addition to the upregulation of ISG15, IFN- $\alpha$ also induces the expression of the E1 activating enzyme UBE1L, the E2 conjugating enzyme UBCH8, and the major E3 ligase HERC5. Therefore, one of the immediate effects of IFN- $\alpha$ is to increase ISGylation through the sequential actions of these three enzymes (29). Although the E1 activating enzyme UBE1L is $45 \%$ homologous to the ubiquitin E1 enzyme (UBE1), it does not affect the ubiquitin level and function, and therefore UBE1L is specific for ISGylation (30). However, the E2 conjugating enzyme UBCH8 and the E3 ligase HERC5 can acquire either ISG15 or ubiquitin as substrates. Therefore, the effect of IFN- $\alpha$ on the E1 enzyme UBE1L may be specific for ISGylation, but its effect on E2 enzyme UBCH8 and E3 ligase HERC5 can affect both ISGylation and ubiquitination. This study demonstrated that the level of ISG15 in HeLa cells can be either induced by IFN- $\alpha$ treatment or can be artificially overexpressed by transfection with a ISG15 overexpression plasmid. The ISG15induced inhibition of HeLa cell proliferation is associated with free ISG15 regardless of ISGylation. Additionally, the anti- and pro-tumor effects of ISG15 and ISGylation have been reported, and it has also been suggested that increased ISGylation and decreased free ISG15 may lead to cancer (31-36). Moreover, it has been indicated that ISG15 exerts an anti-apoptotic effect on pancreatic beta MIN6 cells (37). Thus, free ISG15 is a suppressor of tumorigenesis.

It has been well accepted that p53 is a key factor in tumor suppression and the main activating pathway is DNA damage (38). Activated-p53 in turn induces cell cycle arrest 
and/or apoptosis. IFN-treated cells are more susceptible to p53-dependent apoptosis and HeLa cells undergo apoptosis in response to DNA damage in a p53-dependent manner $(39,40)$. We explored the expression of p53 and its downstream protein p21 in HeLa cells treated with IFN- $\alpha$ or in cells overexpressing ISG15. Consistent with what we have observed previously in HepG2 cells (22), the levels of p53 and p21 were significantly upregulated in HeLa cells when they were either treated with IFN- $\alpha$ or transfected with ISG15 overexpression vector. However, the knockdown of ISG15 significantly attenuated the IFN- $\alpha$-induced increase in p53 expression, suggesting that ISG15 plays an essential role in IFN- $\alpha$-induced p53 expression, as well as apoptosis. The role of ISG15 in this process was also corroborated by the fact that p53 and p 21 expression were upregulated by the overexpression of ISG15. Furthermore, the overexpression of ISG15 not only upregulated p53 and p21, but also promoted HeLa cell apoptosis. However, ISG15induced apoptosis was markedly abrogated when the cells were transfected with siRNA against p53. This demonstrated that ISG15-induced HeLa cell apoptosis is p53-dependent. However, the ISGylation-mediated p53 degradation revealed another pathway regulating p53 stability (41-43). Taken together, these data demonstrate the existence of the IFN- $\alpha /$ ISG15/p53 axis in HeLa cells, and demonstrate that is plays pivotal roles in IFN- $\alpha$-induced cancer cell growth inhibition, as well as apoptotic cell death.

In conclusion, in this study, using HeLa cells as a cancer model system, we explored the roles of ISG15 in IFN- $\alpha$ induced cancer cell growth inhibition and apoptotic cell death. By treating the cells with IFN- $\alpha$ and manipulating the expression of ISG15, we identified the IFN- $\alpha /$ ISG15/p53 axis in HeLa cells. Therefore, any strategies manipulating the expression of ISG15 without IFNs may prove to be effective in the treatment of cervical cancer. Furthermore, given the fact that resistance to IFN- $\alpha$ has often been observed in clinical trails, ISG15 may serve as a novel therapeutic target in the treatment of cervical and possibly, other types of cancer.

\section{Acknowledgements}

This study was supported by the Open-End Fund for the Valuable and Precision Instruments of Central South University and National Basic Research Program of China (grant no. 2011CB910700-704).

\section{References}

1. Duenas-Gonzalez A, Serrano-Olvera A, Cetina L and Coronel J: New molecular targets against cervical cancer. Int J Womens Health 6: 1023-1031, 2014

2. Dasari S, Wudayagiri R and Valluru L: Cervical cancer: biomarkers for diagnosis and treatment. Clin Chim Acta 445: 7-11, 2015.

3. Schiffman MH: Recent progress in defining the epidemiology of human papillomavirus infection and cervical neoplasia. J Natl Cancer Inst 84: 394-398, 1992.

4. Massad LS, Turyk ME, Bitterman P and Wilbanks GD Interferon-alpha and all-trans-retinoic acid reversibly inhibit the in vitro proliferation of cell lines derived from cervical cancers. Gynecol Oncol 60: 428-434, 1996.

5. Kim KY, Blatt L and Taylor MW: The effects of interferon on the expression of human papillomavirus oncogenes. J Gen Virol 81 695-700, 2000

6. Koromilas AE, Li S and Matlashewski G: Control of interferon signaling in human papillomavirus infection. Cytokine Growth Factor Rev 12: 157-170, 2001.
7. Barnard P, Payne E and McMillan NA: The human papillomavirus E7 protein is able to inhibit the antiviral and anti-growth functions of interferon- $\alpha$. Virology 277: 411-419, 2000.

8. Lenschow DJ,Lai C, Frias-Staheli N, Giannakopoulos NV, Lutz A Wolff T, Osiak A, Levine B, Schmidt RE, García-Sastre A, et al: IFN-stimulated gene 15 functions as a critical antiviral molecule against influenza, herpes, and Sindbis viruses. Proc Natl Acad Sci USA 104: 1371-1376, 2007.

9. Friedman RM: Clinical uses of interferons. Br J Clin Pharmacol 65: 158-162, 2008.

10. Sadler AJ and Williams BR: Interferon-inducible antiviral effectors. Nat Rev Immunol 8: 559-568, 2008.

11. Zhang T, Sun HC, Zhou HY, Luo JT, Zhang BL, Wang P, Wang L, Qin LX, Ren N, Ye SL, et al: Interferon alpha inhibits hepatocellular carcinoma growth through inducing apoptosis and interfering with adhesion of tumor endothelial cells. Cancer Lett 290: 204-210, 2010.

12. Shi WY, Cao C and Liu L: Interferon $\alpha$ induces the apoptosis of cervical cancer HeLa cells by activating both the intrinsic mitochondrial pathway and endoplasmic reticulum stress-induced pathway. Int J Mol Sci 17: pii: E1832, 2016.

13. Kim KI, Yan M, Malakhova O, Luo JK, Shen MF, Zou W, de la Torre JC and Zhang DE: Ube1L and protein ISGylation are not essential for alpha/beta interferon signaling. Mol Cell Biol 26: 472-479, 2006.

14. Hishiki T, Han Q, Arimoto K, Shimotohno K, Igarashi T, Vasudevan SG, Suzuki Y and Yamamoto N: Interferon-mediated ISG15 conjugation restricts dengue virus 2 replication. Biochem Biophys Res Commun 448: 95-100, 2014.

15. Loeb KR and Haas AL: The interferon-inducible 15-kDa ubiquitin homolog conjugates to intracellular proteins. J Biol Chem 267: 7806-7813, 1992.

16. Haas AL, Ahrens P, Bright PM and Ankel H: Interferon induces a 15-kilodalton protein exhibiting marked homology to ubiquitin. J Biol Chem 262: 11315-11323, 1987.

17. Darb-Esfahani S, Sinn BV, Rudl M, Sehouli J, Braicu I, Dietel M and Denkert C: Interferon-stimulated gene, $15 \mathrm{kDa}$ (ISG15) in ovarian high-grade serous carcinoma: prognostic impact and link to NF- $\mathrm{BB}$ pathway. Int J Gynecol Pathol 33: 16-22, 2014.

18. Jeon YJ, Yoo HM and Chung CH: ISG15 and immune diseases. Biochim Biophys Acta 1802: 485-496, 2010.

19. Burks J, Reed RE and Desai SD: Free ISG15 triggers an antitumor immune response against breast cancer: a new perspective. Oncotarget 6: 7221-7231, 2015.

20. Zhao C, Hsiang TY, Kuo RL and Krug RM: ISG15 conjugation system targets the viral NS1 protein in influenza A virus-infected cells. Proc Natl Acad Sci USA 107: 2253-2258, 2010.

21. Jeon YJ, Jo MG, Yoo HM, Hong SH, Park JM, Ka SH, Oh KH, Seol JH, Jung YK and Chung CH: Chemosensitivity is controlled by p63 modification with ubiquitin-like protein ISG15. J Clin Invest 122: 2622-2636, 2012.

22. Wan XX, Chen HC, Khan MA, Xu AH, Yang FL, Zhang YY and Zhang DZ: ISG15 inhibits IFN- $\alpha$-resistant liver cancer cell growth. BioMed Res Int 2013: 570909, 2013.

23. Strander $\mathrm{H}$ and Einhorn S: Interferons and the tumor cell. Biotherapy 8: 213-218, 1996.

24. Pfeffer LM, Dinarello CA, Herberman RB, Williams BR, Borden EC, Bordens R, Walter MR, Nagabhushan TL, Trotta PP and Pestka S: Biological properties of recombinant alpha-interferons: 40th anniversary of the discovery of interferons. Cancer Res 58: 2489-2499, 1998.

25. Darnell JE Jr, Kerr IM and Stark GR: Jak-STAT pathways and transcriptional activation in response to IFNs and other extracellular signaling proteins. Science 264: 1415-1421, 1994.

26. O'Shea JJ, Gadina M and Schreiber RD: Cytokine signaling in 2002: new surprises in the Jak/Stat pathway. Cell 109 (Suppl): S121-S131, 2002

27. Sainz B Jr, Martín B, Tatari M, Heeschen C and Guerra S: ISG15 is a critical microenvironmental factor for pancreatic cancer stem cells. Cancer Res 74: 7309-7320, 2014.

28. Takaoka A, Hayakawa S, Yanai H, Stoiber D, Negishi H, Kikuchi H, Sasaki S, Imai K, Shibue T, Honda K and Taniguchi T: Integration of interferon- $\alpha / \beta$ signalling to 53 responses in tumour suppression and antiviral defence. Nature 424: 516-523, 2003.

29. Malakhov MP, Malakhova OA, Kim KI, Ritchie KJ and Zhang DE: UBP43 (USP18) specifically removes ISG15 from conjugated proteins. J Biol Chem 277: 9976-9981, 2002.

30. Yuan W and Krug RM: Influenza B virus NS1 protein inhibits conjugation of the interferon (IFN)-induced ubiquitin-like ISG15 protein. EMBO J 20: 362-371, 2001. 
31. D'Cunha J, Knight E Jr, Haas AL, Truitt RL and Borden EC: Immunoregulatory properties of ISG15, an interferon-induced cytokine. Proc Natl Acad Sci USA 93: 211-215, 1996.

32. Owhashi M, Taoka Y, Ishii K, Nakazawa S, Uemura H and Kambara H: Identification of a ubiquitin family protein as a novel neutrophil chemotactic factor. Biochem Biophys Res Commun 309: 533-539, 2003.

33. Campbell JA and Lenschow DJ: Emerging roles for immunomodulatory functions of free ISG15. J Interferon Cytokine Res 33: 728-738, 2013.

34. Desai SD, Reed RE, Burks J, Wood LM, Pullikuth AK, Haas AL, Liu LF, Breslin JW, Meiners S and Sankar S: ISG15 disrupts cytoskeletal architecture and promotes motility in human breast cancer cells. Exp Biol Med (Maywood) 237: 38-49, 2012.

35. Burks J, Reed RE and Desai SD: ISGylation governs the oncogenic function of Ki-Ras in breast cancer. Oncogene 33: 794-803, 2014.

36. Kiessling A, Hogrefe C, Erb S, Bobach C, Fuessel S, Wessjohann L and Seliger B: Expression, regulation and function of the ISGylation system in prostate cancer. Oncogene 28: 2606-2620, 2009.

37. Yoshikawa A, Imagawa A, Nakata S, Fukui K, Kuroda Y, Miyata Y, Sato Y, Hanafusa T, Matsuoka TA, Kaneto H, et al: Interferon stimulated gene 15 has an anti-apoptotic effect on MIN6 cells. Endocr J 61: 883-890, 2014.
38. Butz K, Shahabeddin L, Geisen C, Spitkovsky D, Ullmann A and Hoppe-Seyler F: Functional p53 protein in human papillomavirus-positive cancer cells. Oncogene 10: 927-936, 1995

39. Mendonca MS, Howard KL, Farrington DL, Desmond LA, Temples TM, Mayhugh BM, Pink JJ and Boothman DA: Delayed apoptotic responses associated with radiation-induced neoplastic transformation of human hybrid cells. Cancer Res 59: 3972-3979, 1999

40. Yang J, Dai LX, Chen M, Li B, Ding N, Li G, Liu YQ, Li MY, Wang BN, Shi XL and Tan HB: Inhibition of antiviral drug cidofovir on proliferation of human papillomavirus-infected cervical cancer cells. Exp Ther Med 12: 2965-2973, 2016.

41. Huang YF, Wee S, Gunaratne J, Lane DP and Bulavin DV: Isg15 controls p53 stability and functions. Cell Cycle 13: 2200-2210,2014.

42. Huang YF and Bulavin DV: Oncogene-mediated regulation of p53 ISGylation and functions. Oncotarget 5: 5808-5818, 2014.

43. Menendez D and Anderson CW: p53 vs. ISG15: stop, you're killing me. Cell Cycle 13: 2160-2161, 2014. 